\title{
PROPOSTA DE FABRICAÇÃO DE ARMAÇÃO DE ÓCULOS ATRAVÉS DA IMPRESSÃO 3D: UMA ABORDAGEM A PARTIR DO MÉTODO AHP-GAUSSIANO
}

\author{
Brayan de Almeida Lima (UNIGRANRIO) brayan_lima@hotmail.com.br \\ Marcos dos Santos (IME) marcosdossantos@ime.eb.br \\ Daniel Augusto de Moura Pereira (UFCG) danielmoura@ufcg.edu.br \\ Rubens Lopes de Oliveira (UFRJ) rubensli@ pep.ufrj.br
}

\section{Resumo}

A utilização da tecnologia de manufatura aditiva, popularmente chamada impressão 3D, está cada vez mais presente na vida das pessoas. O objetivo deste trabalho é propor uma análise multicritério, através de um método de apoio à tomada de decisão, na seleção de um material utilizado para impressão 3D, que se enquadre nos critérios julgados mais relevantes, na confecção de armações de óculos, onde se espera um possível menor custo do que o método tradicional de aquisição, em óticas, por exemplo. $\mathrm{O}$ método escolhido para se realizar o processo decisório é o AHP-Gaussiano, uma variação do método AHP tradicional. A partir da análise multicritério, o material selecionado foi o Nylon, material este, altamente resistente à impactos, parcialmente flexível e que resiste a abrasão, além do custo não ser tão elevado, o que faz com que seja uma boa escolha. O pensamento da confecção destas armações, a partir da impressão 3D, é poder levar à sociedade uma maneira mais barata e com qualidade de adquiri-las, cujo objetivo é o uso com lentes de grau, pois sua ausência, quando há necessidade de uso, por relacionar-se diretamente com a saúde pública, pode ocasionar danos a visão, como estresse, miopia, hipermetropia, astigmatismo, entre outros.

Palavras-Chaves: Tomada de decisão, AHP-Gaussiano, Manufatura aditiva, Impressão 3D, Armação de óculos.

\section{Introdução}

O mundo vem sofrendo positivamente com o desenvolvimento da CT\&I desde o século XVIII, a percorrer pelas três Revoluções Industriais, para mudar a percepção humana nos hábitos e costumes sobre a produção de bens e serviços e no âmbito científico e tecnológico. (MATTOS; GUIMARÃES, 2012, p.3).

A atual revolução em que o mundo está vivenciando, a Indústria 4.0, mostra as mudanças mais significativas tanto na área tecnológica quanto na inovação, gerando um cenário competitivo a partir destas inovações tecnológicas. (ALMEIDA, 2019) 
A Manufatura Aditiva, popularmente chamada impressão 3D, um dos pilares da Indústria 4.0, foi inventada pelo estadunidense Chuck Hull no ano de 1983, que desenvolveu a estereolitografia, antecessora a impressão 3D que conhecemos atualmente. (SACOMANO; et al., 2018)

No Brasil, a Manufatura Aditiva teve seu início no ano de 1998, pelo designer brasileiro Maurício Klabin pioneiro na utilização da tecnologia de impressão 3D. Nos anos 2000, a impressão 3D foi muito utilizada para a criação de joias, pelo brasileiro Antônio Bernardo, um dos pioneiros a utilizar a tecnologia para desenvolver anéis impressos em 3D com cera para fundição. (VOLPATO, 2017)

As impressoras 3D, na área de prototipagem rápida, estão cada vez mais presentes nas indústrias. Geralmente, o processo de Manufatura Aditiva já possui um rumo definido no ambiente de produção industrial por estar se consolidando com o passar dos anos, consequentemente, devido ao avanço desta tecnologia, o que gera redução dos custos de produção, como redução dos valores de aquisição dos equipamentos e da matéria-prima, assim como surgimento de mão de obra especializada. (MEBOLDT; KLAHN, 2017, p.329).

Neste sentido, Leary (2019) aponta os principais benefícios da Manufatura Aditiva (MA) podem ser definidos em comparação com as características tecnológicas da fabricação tradicional. Em resumo, os benefícios podem ser marcados por: redução dos custos de produção; maior complexidade do produto; materiais personalizados; design generativo; redução dos desperdícios; e distribuição da fabricação (projeto).

A tecnologia de impressão 3D está associada com a prototipagem rápida e a confecção de peças ou produtos. Entende-se que esta tecnologia é uma grande facilitadora na obtenção da redução de custos, redução de desperdícios, maior complexidade geométrica aplicada, entre outros requisitos que fazem dela um bom meio de fabricação.

Com o passar dos anos a tecnologia de impressão 3D vem sofrendo constantes atualizações para tornar acessível a obtenção de equipamentos e principalmente materiais de impressão. Os avanços nos estudos sobre os materiais que podem ser empregados no processo possibilita termos um custo reduzido comparado aos anos iniciais do uso de tal tecnologia. Hoje temos uma grande diversificação destes materiais, desde ABS até Fibra de carbono, por exemplo.

Selecionar um dentre inúmeras opções de materiais torna-se um processo complexo quando de forma intuitiva, pois pode ser que o resultado não seja o melhor, para isto, de forma racional, é preferível a utilização de métodos de apoio a tomada de decisão multicritério. 
Diante deste cenário, como é possível ser mais assertivo no processo de tomada de decisão, empregando uma série de critérios, na seleção do melhor material utilizável na Manufatura Aditiva, para a fabricação de armações de óculos?

Dado questionamento, acima, foi debatido e escolhido o método de Apoio à Tomada de Decisão Multicritério AHP-Gaussiano, para auxiliar na seleção da melhor alternativa a ser empregada na fabricação de armações de óculos.

Logo, o objetivo deste trabalho, é através do método de Apoio à Tomada de Decisão Multicritério AHP-Gaussiano, selecionar um material utilizado para impressão 3D, a partir de determinados critérios julgados de maior relevância para os autores, para utilização na manufatura de armações de óculos.

\section{Fundamentação teórica}

\subsection{Tomada de decisão}

A tomada de decisão é realizada sempre que há a necessidade de uma solução para um determinado problema. Mesmo que exista uma única solução, a alternativa pode ser realizar ou não realizar tal ação. Direcionar o foco a um problema, viabiliza a possibilidade de direcionar todos os esforços corretamente ao processo. (GOMES; GOMES, 2019, p.1)

O processo decisório pode conceber-se através de um único decisor, ou um grupo de decisores, partindo do princípio de alcançar o melhor resultado para um determinado problema. (GOMES; GOMES, 2019, p.1)

\subsubsection{AHP-Gaussiano}

O método multicritério Analytic Hierarchy Process - Gaussian (AHP-Gaussian), foi introduzido por Santos, Costa e Gomes (2021), onde visa uma nova abordagem ao método original AHP, que se baseia em uma análise de sensibilidade proveniente do fator Gaussiano. Com essa abordagem é possível obter os pesos dos atributos a partir de inputs quantitativos das alternativas em seus respectivos atributos, a partir dos dados inseridos na matriz de decisão. (SANTOS; COSTA; GOMES, 2021)

O método visa acabar com a dependência que existe da matriz de avaliação entre os critérios, eliminando a necessidade de avaliação par a par entre os atributos na obtenção dos seus 
respectivos pesos, onde os cálculos incluem a média e o desvio padrão para a alcance da ordenação das alternativas. O AHP-Gaussiano pertence aos métodos compensatórios, os atributos são independentes e os atributos qualitativos são convertidos em atributos quantitativos. (SANTOS; COSTA; GOMES, 2021)

Etapas do método AHP-Gaussiano:

- A primeira etapa do método é estabelecer a matriz de decisão, com as alternativas e critérios a serem julgados no processo decisório;

- A segunda etapa é calcular a média das alternativas;

- A terceira etapa é calcular o desvio padrão de cada alternativa para cada critério;

- A quarta etapa é calcular o fator Gaussiano para cada critério;

- A quinta etapa é multiplicar o fator Gaussiano pela matriz de decisão

- A sexta etapa consiste na normalização dos resultados; e

- Na sétima etapa é obtido a ordenação das alternativas.

\subsection{Manufatura aditiva}

A Manufatura Aditiva é um processo produtivo que envolve a fabricação de peças e componentes, até mesmo produtos inteiros, a partir de sobrepostas de material, em geral, provenientes de cartuchos plásticos em filamentos de secção circular, onde ocorre o aquecimento destes no cabeçote da impressora 3D, onde são injetadas diversas camadas para serem alcançadas as dimensões previamente programadas e modeladas em um software de desenho 3D, Computer Aided Design (CAD), e enviado ao software de programação da impressora 3D, sendo eles, acompanhado por um hardware. (ALMEIDA, Paulo, 2019)

\section{Metodologia}

\subsection{Questões de pesquisa}

Dentro das limitações visualizadas, para a realização deste estudo de caso se fez necessário a redução do número de alternativas e atributos, ocorrendo uma pré-seleção. Por existir uma grande diversidade de materiais e características, em comum acordo entre os autores, foram considerados apenas os de maior impacto para o objeto de estudo. 


\subsection{Seleção dos participantes e caso estudado}

Os participantes são os próprios autores deste trabalho. Quanto ao direcionamento, fica por conta da fabricação de armações de óculos em impressão 3D, com intuito de exaltar as melhores alternativas e suas características.

\subsection{Procedimentos de coleta}

A coleta das informações foi realizada a partir de documentos abertos e publicações via internet, jornais e revistas, com alta visibilidade e credibilidade no assunto e considerados importantes, fontes nacionais e internacionais, como os sites Ultimaker e Simplify3D, especializados em conteúdo sobre impressão 3D.

\subsection{Procedimento de análise de dados}

Os dados e as informações coletadas foram analisadas de forma qualitativa e quantitativa de modo a expor todos os fatores que contribuem para a solução da situação-problema. O tratamento e análise dos dados e das informações ocorreram através de planilhas e Tabelas.

\subsection{Procedimento de validação}

O processo decisório foi realizado através do software MS Excel, onde são introduzidas as informações dos materiais e seus respectivos atributos. A decisão de possuir apenas um decisor deve-se ao fato de não haver a subjetividade sobre as informações, já que os dados técnicos são expostos pelos fabricantes e pesquisadores. O software gera os resultados que são validados pelo decisor. Além do MS Exel, foi utilizado o Gaussian AHP Software Web (v.1) para confirmar os resultados obtidos.

\section{Resultados e Discussão}

\subsection{Seleção do material}

A seleção dos materiais, para a fabricação das armações de óculos, considerou uma sucessão de critérios pertinentes para uma boa qualidade do produto, tanto em custo, resistência e acabamento (visual). Os dados inseridos na matriz de decisão referentes as alternativas e aos critérios foram retirados do site Simplify3D (2021). 
Para as alternativas, foram pré-selecionados os materiais: ABS (A1); Flexible (A2); PLA (A3); PETG (A4); Nylon (A5); Carbon Fiber Filled (A6); Polycarbonate (A7); e Polypropylene (A8).

Para os critérios, foram pré-selecionados: Ultimate Strength (C1); Stiffness (C2); Durability (C3); Maximum Service Temperature (C4); Coefficient of Thermal Expansion (C5); Density (C6); Price (per kg) (C7); Printability (C8); Extruder Temperature (C9); Bed temperature (C10); Flexible (C11); Impact Resistant (C12); Heat Resistant (C13); Fatigue Resistant (C14); e Heated Bed Not Required (C15).

\subsubsection{Aplicação da tomada de decisão multicritério}

De maneira a seguir os passos expressos no item 4.1.1. AHP-Gaussiano, a primeira etapa será a criação da matriz de decisão onde serão inseridos os dados de cada alternativa em seus respectivos critérios.

Tabela 1 - Matriz de decisão

\begin{tabular}{ccccccccc}
\hline $\mathbf{-}$ & $\mathbf{A 1}$ & $\mathbf{A 2}$ & $\mathbf{A 3}$ & $\mathbf{A 4}$ & $\mathbf{A 5}$ & $\mathbf{A 6}$ & $\mathbf{A 7}$ & $\mathbf{A 8}$ \\
\hline $\mathbf{C 1}$ & $40 \mathrm{MPa}$ & $43 \mathrm{MPa}$ & $65 \mathrm{MPa}$ & $53 \mathrm{MPa}$ & $85 \mathrm{MPa}$ & $48 \mathrm{MPa}$ & $72 \mathrm{MPa}$ & $32 \mathrm{MPa}$ \\
\hline $\mathbf{C 2}$ & 5 & 1 & 7,5 & 5 & 5 & 10 & 6 & 4 \\
\hline $\mathbf{C 3}$ & 8 & 9 & 4 & 8 & 10 & 3 & 10 & 9 \\
\hline $\mathbf{C 4}$ & $98^{\circ} \mathrm{C}$ & $74^{\circ} \mathrm{C}$ & $52^{\circ} \mathrm{C}$ & $73^{\circ} \mathrm{C}$ & $95^{\circ} \mathrm{C}$ & $52^{\circ} \mathrm{C}$ & $121^{\circ} \mathrm{C}$ & $100^{\circ} \mathrm{C}$ \\
\hline $\mathbf{C 5}$ & 90 & 157 & 68 & 60 & 95 & 57.5 & 69 & 150 \\
\hline
\end{tabular}




\begin{tabular}{lcccccccc}
\hline$\mu \mathrm{m} / \mathrm{m}-{ }^{\circ} \mathrm{C}$ & $\mu \mathrm{m} / \mathrm{m}-{ }^{\circ} \mathrm{C}$ & $\mu \mathrm{m} / \mathrm{m}-{ }^{\circ} \mathrm{C}$ & $\mu \mathrm{m} / \mathrm{m}-{ }^{\circ} \mathrm{C}$ & $\mu \mathrm{m} / \mathrm{m}-{ }^{\circ} \mathrm{C}$ & $\mu \mathrm{m} / \mathrm{m}-{ }^{\circ} \mathrm{C}$ & $\mu \mathrm{m} / \mathrm{m}-{ }^{\circ} \mathrm{C}$ & $\mu \mathrm{m} / \mathrm{m}-{ }^{\circ} \mathrm{C}$ \\
\hline C6 & $1.04 \mathrm{~g} / \mathrm{cm}^{3}$ & $1.23 \mathrm{~g} / \mathrm{cm}^{3}$ & $1.24 \mathrm{~g} / \mathrm{cm}^{3}$ & $1.23 \mathrm{~g} / \mathrm{cm}^{3}$ & $1.14 \mathrm{~g} / \mathrm{cm}^{3}$ & $1.3 \mathrm{~g} / \mathrm{cm}^{3}$ & $1.2 \mathrm{~g} / \mathrm{cm}^{3}$ & $0.9 \mathrm{~g} / \mathrm{cm}^{3}$ \\
\hline C7 & $\$ 25$ & $\$ 50$ & $\$ 25$ & $\$ 40$ & $\$ 45$ & $\$ 55$ & $\$ 57,5$ & $\$ 90$ \\
\hline C8 & 8 & 6 & 9 & 9 & 8 & 8 & 6 & 4 \\
\hline C9 & $250^{\circ} \mathrm{C}$ & $245^{\circ} \mathrm{C}$ & $220^{\circ} \mathrm{C}$ & $250^{\circ} \mathrm{C}$ & $270^{\circ} \mathrm{C}$ & $230^{\circ} \mathrm{C}$ & $310^{\circ} \mathrm{C}$ & $250^{\circ} \mathrm{C}$ \\
\hline $\mathbf{C 1 0}$ & $110^{\circ} \mathrm{C}$ & $60^{\circ} \mathrm{C}$ & $60^{\circ} \mathrm{C}$ & $90^{\circ} \mathrm{C}$ & $90^{\circ} \mathrm{C}$ & $60^{\circ} \mathrm{C}$ & $120^{\circ} \mathrm{C}$ & $100^{\circ} \mathrm{C}$ \\
\hline $\mathbf{C 1 1}$ & 0 & 1 & 0 & 0 & 1 & 0 & 0 & 1 \\
\hline $\mathbf{C 1 2}$ & 1 & 0 & 0 & 0 & 1 & 0 & 1 & 0 \\
\hline $\mathbf{C 1 3}$ & 1 & 0 & 0 & 0 & 1 & 0 & 1 & 1 \\
\hline $\mathbf{C 1 4}$ & 0 & 1 & 0 & 1 & 1 & 0 & 1 & 1 \\
\hline $\mathbf{C 1 5}$ & 0 & 1 & 1 & 0 & 0 & 1 & 0 & 0 \\
\hline
\end{tabular}

Fonte: Autores (2021)

A próxima etapa é realizar a normalização da matriz de decisão, Tabela 1, acima, cujo objetivo é fazer com que os valores de cada atributo em cada alternativa, assumam valores padronizados aos demais, ou seja, acabando com as diferentes unidades de medidas que cada atributo proporciona.

Antes da normalização, foi realizado o tratamento dos dados, Tabela 2.

Tabela 2 - Tratamento da matriz de decisão

\begin{tabular}{cccccccccc}
\hline - & - & $\mathbf{A 1}$ & $\mathbf{A 2}$ & $\mathbf{A 3}$ & $\mathbf{A 4}$ & $\mathbf{A 5}$ & $\mathbf{A 6}$ & $\mathbf{A 7}$ & $\mathbf{A 8}$ \\
\hline MAX & $\mathbf{C 1}$ & 40 & 43 & 65 & 53 & 85 & 48 & 72 & 32 \\
\hline MAX & $\mathbf{C 2}$ & 5 & 1 & 7,5 & 5 & 5 & 10 & 6 & 4 \\
\hline MAX & $\mathbf{C 3}$ & 8 & 9 & 4 & 8 & 10 & 3 & 10 & 9 \\
\hline MIN & $\mathbf{C 4}$ & 98 & 74 & 52 & 73 & 95 & 52 & 121 & 100 \\
\hline MIN & $\mathbf{C 5}$ & 90 & 157 & 68 & 60 & 95 & 57,5 & 69 & 150 \\
\hline MAX & $\mathbf{C 6}$ & 1,04 & 1,23 & 1,24 & 1,23 & 1,14 & 1,3 & 1,2 & 0,9 \\
\hline MIN & $\mathbf{C 7}$ & 25 & 50 & 25 & 40 & 45 & 55 & 57,5 & 90 \\
\hline MAX & $\mathbf{C 8}$ & 8 & 6 & 9 & 9 & 8 & 8 & 6 & 4 \\
\hline MIN & $\mathbf{C 9}$ & 250 & 245 & 220 & 250 & 270 & 230 & 310 & 250 \\
\hline MIN & $\mathbf{C 1 0}$ & 110 & 60 & 60 & 90 & 90 & 60 & 120 & 100 \\
\hline MAX & C11 & 0 & 1 & 0 & 0 & 1 & 0 & 0 & 1 \\
\hline MAX & $\mathbf{C 1 2}$ & 1 & 0 & 0 & 0 & 1 & 0 & 1 & 0 \\
\hline MAX & $\mathbf{C 1 3}$ & 1 & 0 & 0 & 0 & 1 & 0 & 1 & 1 \\
\hline MAX & $\mathbf{C 1 4}$ & 0 & 1 & 0 & 1 & 1 & 0 & 1 & 1 \\
\hline MAX & $\mathbf{C 1 5}$ & 0 & 1 & 1 & 0 & 0 & 1 & 0 & 0 \\
\hline & & & & Fonte: Autores $(2021)$ & & & &
\end{tabular}

A normalização segue, para valores que se queira maximizar, monotônico de lucro, utiliza-se a fórmula (1). 


$$
N=\frac{a_{i j}}{\sum a_{i j}}(1)
$$

Para valores que se queira minimizar, monotônico de custo, utiliza-se a fórmula (2).

$$
N=\frac{\left(\frac{1}{a_{i j}}\right)}{\left(\frac{a_{i j}}{\sum a_{i j}}\right)}(2)
$$

Tabela 2 - Matriz de decisão normalizada

\begin{tabular}{cccccccccc}
\hline - & - & A1 & A2 & A3 & A4 & A5 & A6 & A7 & A8 \\
\hline MAX & C1 & 0,091 & 0,098 & 0,148 & 0,121 & 0,194 & 0,110 & 0,164 & 0,073 \\
\hline MAX & C2 & 0,115 & 0,023 & 0,172 & 0,115 & 0,115 & 0,230 & 0,138 & 0,092 \\
\hline MAX & C3 & 0,131 & 0,148 & 0,066 & 0,131 & 0,164 & 0,049 & 0,164 & 0,148 \\
\hline MIN & C4 & 0,097 & 0,129 & 0,184 & 0,131 & 0,101 & 0,184 & 0,079 & 0,096 \\
\hline MIN & C5 & 0,113 & 0,065 & 0,150 & 0,170 & 0,107 & 0,178 & 0,148 & 0,068 \\
\hline MAX & C6 & 0,112 & 0,133 & 0,134 & 0,133 & 0,123 & 0,140 & 0,129 & 0,097 \\
\hline MIN & C7 & 0,206 & 0,103 & 0,206 & 0,129 & 0,115 & 0,094 & 0,090 & 0,057 \\
\hline MAX & C8 & 0,138 & 0,103 & 0,155 & 0,155 & 0,138 & 0,138 & 0,103 & 0,069 \\
\hline MIN & C9 & 0,125 & 0,128 & 0,142 & 0,125 & 0,116 & 0,136 & 0,101 & 0,125 \\
\hline MIN & C10 & 0,091 & 0,167 & 0,167 & 0,112 & 0,112 & 0,167 & 0,084 & 0,100 \\
\hline MAX & C11 & 0,000 & 0,333 & 0,000 & 0,000 & 0,333 & 0,000 & 0,000 & 0,333 \\
\hline MAX & C12 & 0,333 & 0,000 & 0,000 & 0,000 & 0,333 & 0,000 & 0,333 & 0,000 \\
\hline MAX & C13 & 0,250 & 0,000 & 0,000 & 0,000 & 0,250 & 0,000 & 0,250 & 0,250 \\
\hline MAX & C14 & 0,000 & 0,200 & 0,000 & 0,200 & 0,200 & 0,000 & 0,200 & 0,200 \\
\hline MAX & C15 & 0,000 & 0,333 & 0,333 & 0,000 & 0,000 & 0,333 & 0,000 & 0,000 \\
\hline
\end{tabular}

Após realizar a normalização, passa-se para a etapa de cálculo da média, desvio padrão e do fator Gaussiano, e a normalização do fator Gaussiano.

Tabela 3 - Etapas 2, 3 e 4

\begin{tabular}{cccccc}
\hline - & - & Média & Desvio Padrão & Fator Gaussiano & $\begin{array}{c}\text { Fator Gaussiano } \\
\text { Normalizado }\end{array}$ \\
\hline MAX & C1 & 0,125 & 0,041 & 0,327 & 0,036 \\
\hline MAX & C2 & 0,125 & 0,060 & 0,480 & 0,053 \\
\hline
\end{tabular}




\begin{tabular}{llllll}
\hline MAX & $\mathbf{C 3}$ & 0,125 & 0,044 & 0,350 & 0,039 \\
\hline MIN & $\mathbf{C 4}$ & 0,125 & 0,040 & 0,321 & 0,036 \\
\hline MIN & $\mathbf{C 5}$ & 0,125 & 0,043 & 0,348 & 0,038 \\
\hline MAX & $\mathbf{C 6}$ & 0,125 & 0,014 & 0,113 & 0,012 \\
\hline MIN & $\mathbf{C 7}$ & 0,125 & 0,054 & 0,434 & 0,048 \\
\hline MAX & $\mathbf{C 8}$ & 0,125 & 0,030 & 0,242 & 0,027 \\
\hline MIN & $\mathbf{C 9}$ & 0,125 & 0,012 & 0,100 & 0,011 \\
\hline MIN & $\mathbf{C 1 0}$ & 0,125 & 0,036 & 0,290 & 0,032 \\
\hline MAX & $\mathbf{C 1 1}$ & 0,125 & 0,173 & 1,380 & 0,153 \\
\hline MAX & $\mathbf{C 1 2}$ & 0,125 & 0,173 & 1,380 & 0,153 \\
\hline MAX & $\mathbf{C 1 3}$ & 0,125 & 0,134 & 1,069 & 0,118 \\
\hline MAX & $\mathbf{C 1 4}$ & 0,125 & 0,104 & 0,828 & 0,092 \\
\hline MAX & $\mathbf{C 1 5}$ & 0,125 & 0,173 & 1,380 & 0,153 \\
\hline
\end{tabular}

Fonte: Autores (2021)

A normalização do fator Gaussiano ocorre da mesma maneira em que é normalizado os atributos monotônicos de lucro, fórmula (1).

A próxima etapa é realizar a multiplicação do fator Gaussiano com a matriz de decisão normalizada.

Tabela 4 - Matriz de decisão normalizada e ponderada

\begin{tabular}{cccccccccc}
\hline - & $\mathbf{-}$ & $\mathbf{A 1}$ & $\mathbf{A 2}$ & $\mathbf{A 3}$ & $\mathbf{A 4}$ & $\mathbf{A 5}$ & $\mathbf{A 6}$ & $\mathbf{A 7}$ & $\mathbf{A 8}$ \\
\hline MAX & $\mathbf{C 1}$ & 0,003 & 0,004 & 0,005 & 0,004 & 0,007 & 0,004 & 0,006 & 0,003 \\
\hline MAX & $\mathbf{C 2}$ & 0,006 & 0,001 & 0,009 & 0,006 & 0,006 & 0,012 & 0,007 & 0,005 \\
\hline MAX & $\mathbf{C 3}$ & 0,005 & 0,006 & 0,003 & 0,005 & 0,006 & 0,002 & 0,006 & 0,006 \\
\hline MIN & C4 & 0,003 & 0,005 & 0,007 & 0,005 & 0,004 & 0,007 & 0,003 & 0,003 \\
\hline MIN & C5 & 0,004 & 0,003 & 0,006 & 0,007 & 0,004 & 0,007 & 0,006 & 0,003 \\
\hline
\end{tabular}




\begin{tabular}{cccccccccc}
\hline MAX & C6 & 0,001 & 0,002 & 0,002 & 0,002 & 0,002 & 0,002 & 0,002 & 0,001 \\
\hline MIN & C7 & 0,010 & 0,005 & 0,010 & 0,006 & 0,006 & 0,005 & 0,004 & 0,003 \\
\hline MAX & C8 & 0,004 & 0,003 & 0,004 & 0,004 & 0,004 & 0,004 & 0,003 & 0,002 \\
\hline MIN & C9 & 0,001 & 0,001 & 0,002 & 0,001 & 0,001 & 0,002 & 0,001 & 0,001 \\
\hline MIN & C10 & 0,003 & 0,005 & 0,005 & 0,004 & 0,004 & 0,005 & 0,003 & 0,003 \\
\hline MAX & C11 & 0,000 & 0,051 & 0,000 & 0,000 & 0,051 & 0,000 & 0,000 & 0,051 \\
\hline MAX & $\mathbf{C 1 2}$ & 0,051 & 0,000 & 0,000 & 0,000 & 0,051 & 0,000 & 0,051 & 0,000 \\
\hline MAX & $\mathbf{C 1 3}$ & 0,030 & 0,000 & 0,000 & 0,000 & 0,030 & 0,000 & 0,030 & 0,030 \\
\hline MAX & $\mathbf{C 1 4}$ & 0,000 & 0,018 & 0,000 & 0,018 & 0,018 & 0,000 & 0,018 & 0,018 \\
\hline MAX & $\mathbf{C 1 5}$ & 0,000 & 0,051 & 0,051 & 0,000 & 0,000 & 0,051 & 0,000 & 0,000 \\
\hline & & & & Fonte: Autores $(2021)$ & & & &
\end{tabular}

Por fim, na última etapa é realizada a ordenação das alternativas do maior para o menor valor, ordem decrescente, onde a alternativa que apresentar o maior valor é a preferível e o inverso também ocorre, onde a alternativa que apresentar o menor valor considera-se a pior.

Tabela 5 - Ordenação

\begin{tabular}{|c|c|c|c|c|}
\hline- & Alternativa & Valor & Valor (\%) & Ordenação \\
\hline A5 & Nylon & 0,192 & $19,2 \%$ & $1^{\circ}$ \\
\hline A2 & Flexible & 0,154 & $15,4 \%$ & $2^{\circ}$ \\
\hline A7 & Polycarbonate & 0,139 & $13,9 \%$ & $3^{\circ}$ \\
\hline A8 & Polypropylene & 0,128 & $12,8 \%$ & $4^{\circ}$ \\
\hline A1 & ABS & 0,122 & $12,2 \%$ & $5^{\circ}$ \\
\hline A3 & PLA & 0,103 & $10,3 \%$ & $6^{\circ}$ \\
\hline A6 & Carbon Fiber Filled & 0,099 & $9,9 \%$ & $7^{\circ}$ \\
\hline A4 & PETG & 0,062 & $6,2 \%$ & $8^{\circ}$ \\
\hline
\end{tabular}

Fonte: Autores (2021)

Com a ordenação dos resultados, fica evidente a preferência pelo material Nylon, de acordo com o método multicritério AHP-Gaussiano.

\subsection{Viabilidade de produção}

\subsubsection{Custos de produção}

Os custos de produção da armação a partir da Manufatura Aditiva, impressão 3D, utilizando o material escolhido, Nylon, é possível ser calculado com algumas informações bases, de acordo com Tabela 6. 
Tabela 6 - Custos de produção

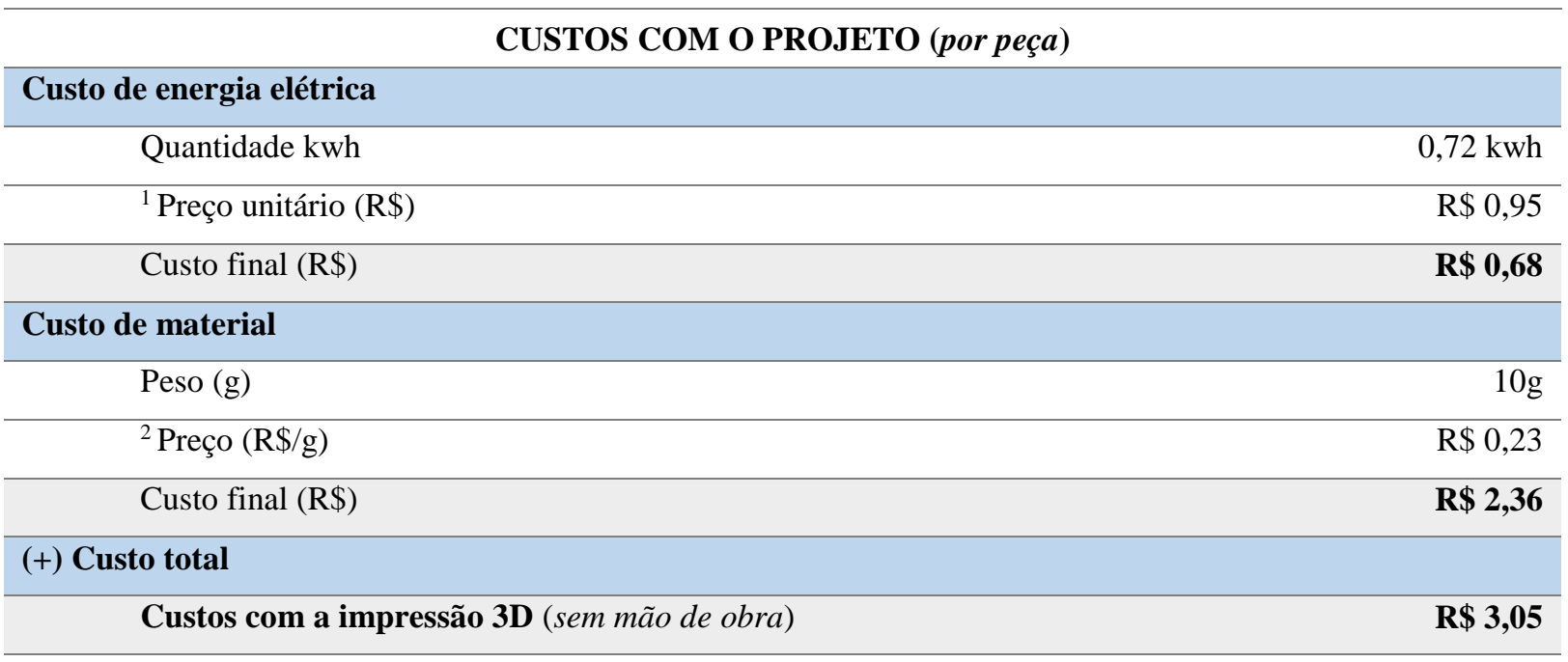

(+) Custo total + mão de obra

\begin{tabular}{lc}
\hline${ }^{3}$ Salário $(\mathrm{R} \$)$ & $\mathbf{R} \mathbf{1 4 , 3 4}$ \\
\hline Custos com a impressão 3D $($ com mão de obra $)$ & $\mathbf{R \$ \mathbf { 1 7 , 3 9 }}$ \\
\hline Tempo de fabricação & $\mathbf{2 h 4 5 m i n}$ \\
\hline Impressão 3D & \\
\hline${ }^{1}$ Preço por kwh, Rio de Janeiro, empresa Light, 2021. & \\
${ }^{2}$ Cotação do dólar R $\$ 5,262$ em 24/08/2021, com conversão de US\$45 para R\$ 236,79. \\
${ }^{3}$ Salário mínimo em 2021 (R \$1.147,00) considerando 220 horas por mês e 2h45min trabalhados. \\
\hline
\end{tabular}
Fonte: Autores (2021)

A utilização do Nylon para impressão 3D na fabricação de armação de óculos apresenta grande viabilidade, com pouco, ou nenhum, desperdício de matéria-prima, a um baixo custo de produção e qualidade acima da média. A vantagem da impressão 3D fica por conta da utilização de poucos recursos, tanto com matéria-prima quanto em equipamento e espaço físico, a desvantagem ocorre no acabamento, necessitando a remoção de rebarbas. Quanto a qualidade, o material Nylon, possui excelentes características de impressão, com um trabalho final robusto, com boa resistência contra quedas, fatigas e ao tempo.

Para se produzir uma armação de baixo custo, podendo beneficiar a população mais carente, que possui a necessidade de uso de óculos de grau, custaria em torno de $\mathrm{R}$ \$, 3,05 a R \$ 17,39 por peça, com possibilidade de se produzir mais de um produto em simultâneo, tornando ainda mais barato a produção (produção em escala). Se for utilizado o ABS ou PLA, o custo de produção seria muito menor, entre $\mathrm{R} \$ 1,99$ a $\mathrm{R} \$ 16,33$.

\subsubsection{Modelagem}


Quanto ao modelo e a modelagem, encontram-se com facilidade design para download em plataformas cujo objetivo é disseminar projetos de impressão 3D, possuindo arquivos pagos e gratuitos, para uso comercial ou não. Com isto, não será nenhum problema obter um mix de produtos ou ainda padronizar um modelo. O modelo de armação de óculos apresentado na Figura 1, foi baixado através da plataforma Thingiverse (2021), de forma gratuita.

\section{Figura 1 - Fatiador Ultimaker Cura}

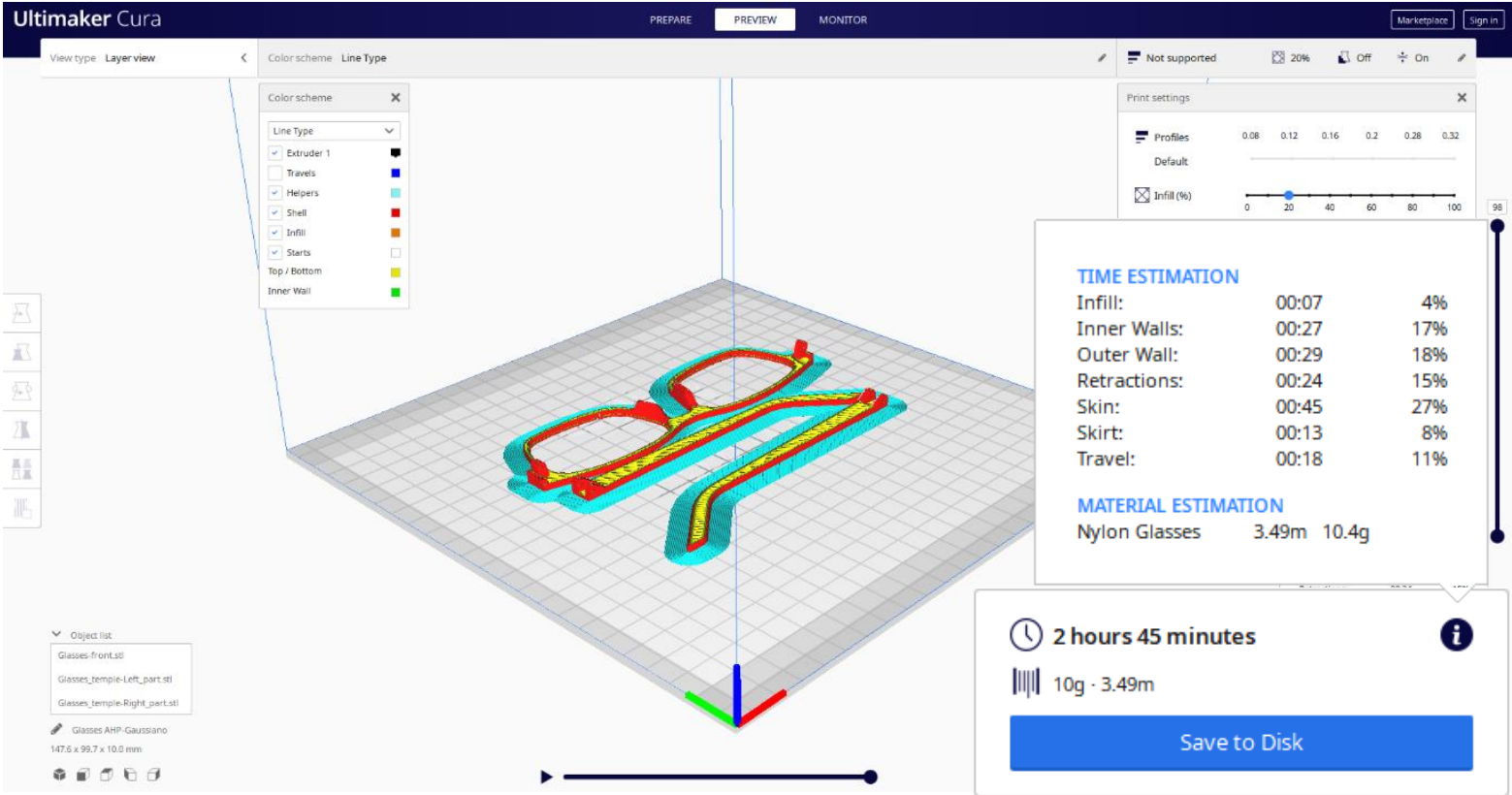

Fonte: Autores (2021)

A modelagem do produto é um processo que requer bastante atenção, as dimensões precisam ser calculadas com precisão, pois, com o modelo padrão definido, serão replicadas diversas cópias e um erro poderá desencadear uma sucessão de eventos desastrosos, podendo perder o produto e necessitando o retrabalho, consequentemente irá gerar aumento dos custos.

\section{Considerações Finais}

O uso do método de apoio a tomada de decisão AHP-Gaussiano foi de grande importância sobre o processo decisório, já que, devido ao excesso de alternativas e atributos, a dificuldade de seleção por um material tornou-se mais complexa.

Os resultados obtidos foram satisfatórios, o Nylon é um material com grande resistência e fácil de realizar a impressão. Seu custo não é tão discrepante quanto aos outros materiais, ficando na média. O acabamento não será um empecilho e o resultado agrada aos autores. 
O material Flexible (Flexível) pode ser considerado um agente de grande interesse, pois possui grande resistência e, também, flexilidade em suas propriedades materiais. Com um custo um pouco maior que o Nylon, porém não tão discrepante.

Tanto o Polycarbonate (Policarbonato) quanto o Polypropylene (Polipropileno), são materiais que podem ser adotados na impressão 3D como possíveis substitutos para o Nylon. Porém, são materiais com o custo muito elevado, por isso o descarte seria iminente.

Já o ABS, o PLA e o PETG podem ser materiais a se pensar na fabricação, mesmo nas últimas colocações do ranking são considerados de grande utilização, já que o custo é bem abaixo da média. São os materiais mais utilizados na impressão 3D, porém sua baixa classificação fica por conta das características dos materiais, como resistência, rigidez e durabilidade. O Carbon Fiber Filled é um excelente material, porém o custo é muito elevado.

Para trabalhos futuros fica a sugestão de utilização de outros métodos para Apoio a Tomada de Decisão para análise de sensibilidade e a impressão 3D do objetivo de estudo.

\section{Referências}

ALMEIDA, Paulo Samuel de. Indústria 4.0: princípios básicos, aplicabilidade e implantação na área industrial. 1. ed. São Paulo: Érica, 2019. 136 p.

GOMES, Luiz Flavio Autran Monteiro; GOMES, Carlos Francisco Simões. Princípios e Métodos para Tomada de Decisão: enfoque multicritério. 6. ed. São Paulo: Atlas, 2019.

LEARY, Martin. Design for Additive Manufacturing: Additive Manufacturing Materials and Technologies. Tradução nossa. [S. I.]: 1. ed. Elsevier, 2019. 358 p.

MATTOS, João Roberto Loureiro de; GUIMARÃES, Leonam dos Santos. Gestão da tecnologia e inovação: uma abordagem prática. 2. ed. São Paulo: Saraiva Uni, 2012. 448 p

MEBOLDT, Mirko; KLAHN, Christoph (ed.). Industrializing Additive Manufacturing: proceedings of additive manufacturing in products and applications. Tradução nossa. 1. ed. Zurique: Springer, 2017. 376 p.

MOREIRA, Miguel Ângelo Lellis; SANTOS, Marcos dos; GOMES, Carlos Francisco Simões. Gaussian AHP Software Web (v.1). 2021.

SANTOS, Marcos dos; COSTA, Igor Pinheiro de Araujo; GOMES, Carlos Francisco Simões. Multicriteria decision-making in the selection of warships: a new approach to the AHP method. International Journal of the Analytic Hierarchy Process, 13(1). 2021. https://doi.org/10.13033/ijahp.v13i1.833.

SACOMANO, José Benedito; et al. Indústria 4.0: conceitos e fundamentos. São Paulo: Blucher, 2018. 169 p

Simplify3D. Filament Properties Table. Disponível em: <https://www.simplify3d.com/support/materialsguide/properties-table/>. Acesso em: 25 de jun. de 2021.

Thingiverse. Glasses. Disponível em: <https://www.thingiverse.com/thing:2798109>. Acesso em: 25 de jun. de 2021.

Ultimaker. Ultimaker Cura. Disponível em: <https://ultimaker.com/>. Acesso em: 25 de jun. de 2021. 
VOLPATO, Neri. Manufatura Aditiva: tecnologias e aplicações da impressão 3D. São Paulo: Blucher, 2017. 